\title{
12. Follow-up Study of the Operative Treatment on the Spinal Cord Tumor and the Chronic Adhesive Arachnoiditis
}

\author{
S. Araki*, N. Tsuyama, M. Nagano, I. Abe*, \\ K. Yamagata** and R. SUZUKI***
}

Sixty-two cases of spinal cord tumor and thirty-nine cases of chronic adhesive arachnoiditis, diagnosed on our operative findings in the past ten years, are reported with statistical considerations. These tumors include thirty-six neurinomas, seven dermoids, five astrocytomas, four meningiomas, four hemangiomas, one glioma, ependymoma, psamoma, neurofibromatosis, cholesteatoma and myxoma. Their myelograms are again compared with the operative findings.

The daily activities as well as physical findings are our main criterion to estimate the long-term results, the longest being twelve years after operation. It revealed that about a third of the patients had returned to their preoperative occupations without any discomfort, while a few had died in the post-operative period and about a half of them, most of whom were with chronic arachnoiditis, were disabled.

* Department of Orthopaedics, Tokyo University School of Medicine.

** Department of Orthopaedics, Tokyo Women's Medical College.

*** Department of Orthopaedics, Tokyo Medical Dental University.

\section{Experimental Histological Studies on the Spinal Injuries}

\section{Kiichi Suzukr, Osamu Natsume, and Atsuo Sugita \\ Urological Clinic, Tohoku University School of Medicine}

The section of the ventral or dorsal horn and the half-side section of the spinal cord in the level of lumbar or sacral segment, which is assumed to be related to the vesical function, was performed on 20 adult dogs to clarify the various functions of the urinary bladder. Thence, the site of lesion and the degeneration of nerve fibers in the spinal cord were examined according to the Marchi's staining for continuous specimens, and furthermore, degeneration in the peripheral nerves was observed.

With the obtained results, the ascending site of the degeneration in the dorsal tract following the spinal lesion was confirmed. In the peripheral nerve with the left half-side section of the lumber segment, degeneration in the $\mathrm{L}_{2}-\mathrm{L}_{4}$ ventral roots, in the inferior splanchnic nerve, in the hypogastric nerve were found, respectively (Table 1 ). It is also noted that almost or all the small- and medium-sized fibers originated from the ventral roots joined into the inferior splanchnic nerves. In the case with half-side section of sacral segment, much 